\title{
A Stochastic Model for Soft Tissue Failure using Acoustic Emission data
}

\author{
D. Sánchez-Molina ${ }^{a *} *$ E. Martínez-González ${ }^{a}$, J. Velázquez-Ameijiøe ${ }^{a}$, \\ J. Llumà ${ }^{a}$, M. C. Rebollo Soria ${ }^{b}$, C. Arregui-Dalmases ${ }^{a}$
}

July 9,2015

${ }^{a}$ UPC, Comte d'Urgell, 187, 08036 Barcelona, Spain 5

${ }^{b}$ IMLC, G.V. Corts Catalanes, 111, 08014 Barcelona, Spain 6

${ }^{*}$ Corresponding author: david.sanchez-molina@upc.edu, Tel. +34 934137335 
can be represented by a non-homogeneous Markov process which is the basis of the stochastic model proposed. This paper shows that a two-parameter model can account for the fiber breaking and the expected distribution for ultimate stress is a Fréchet distribution.

KEYwords: Collagenous Tissues, Acoustic Emission, Stochastic Failure Models, Esophagus.

\section{Introduction}

The non-linear elastic behavior of collagenous soft tissue is well understood, and complex models based on fiber arrangement have been developed [Natali et al., 2009, Kroon \& Holzapfel, 2008]. On the other hand, soft tissue failure shows a much more complex behavior. It is not so well understood and needs additional research [Ionescu et al.,2006]. The experimental data needed to develop models of failure are scarce and the adaptation of existing failure formulations for common engineering materials to soft tissues is difficult because of the micro-structure existing in soft collagenous tissues.

There are different types of soft tissue failure modes. The geometrical description of the last stages of failure has proved to be elusive with high degree of randomness. Reliable failure models is important for some medical procedures such as esophageal dilatation [Fernández-Esparrach et al., 2011] and some other more unusual practical situations involving penetrating trauma injuries. This kind of injuries represents a high socioeconomic cost and represent a significant source of morbidity [Gugala \& Lindsey,2003]

The present work focuses on the understanding of the cumulative effect of internal micro-failures before macroscopic failure. For this purpose a stochastic cumulative damage model based on inhomogeneous renewal-rewarded process is used (an inhomogeneous Markov process measures the number of micro-failures and the "reward" variables control the damage occurred in such a failure). The model presented here differs from other interesting probabilistic models found in the literature [Pradhan \& Chakrabarti, 2003, Kabir et al.,2006] and is innovative in that it provides the distribution of the ultimate stress. On the other hand, another important innovation is the use of Acoustic Emission (AE) to detect experimentally almost all of the relevant micro-failures in soft tissues.

In section 2 the experimental setup is described and the two-parameter 
stochastic model is explained. Section 3 contains the results of the model applied to a human esophagus sample (the distribution for the ultimate stress is computed and the dissipated energy are given). Section 4 discusses the implications of the results.

\section{Methods}

A typical specimen of human esophagus from a donor (PMHS) was used for the experimental work. The age of the PMHS was 63 years-old, and its decease cause was not associated with any esophageal disease (biometric data: male; Body Mass Index, $25.5 \mathrm{~kg} \cdot \mathrm{m}^{-2}$ ). This single sample produced $N$ $=472$ microfailures, which statistically is an adequate medium-size sample. A tensile test was conducted for the sample in order to obtain the stress-strain curve (the acoustic emission measurements and the strain-stress measures of the tensile test were simultaneous). A conventional servo-hydraulic testing machine (microtest EM2/20) was used for the measurement of force. The strain was computed from the data of a camera using motion tracking (see figure 1).
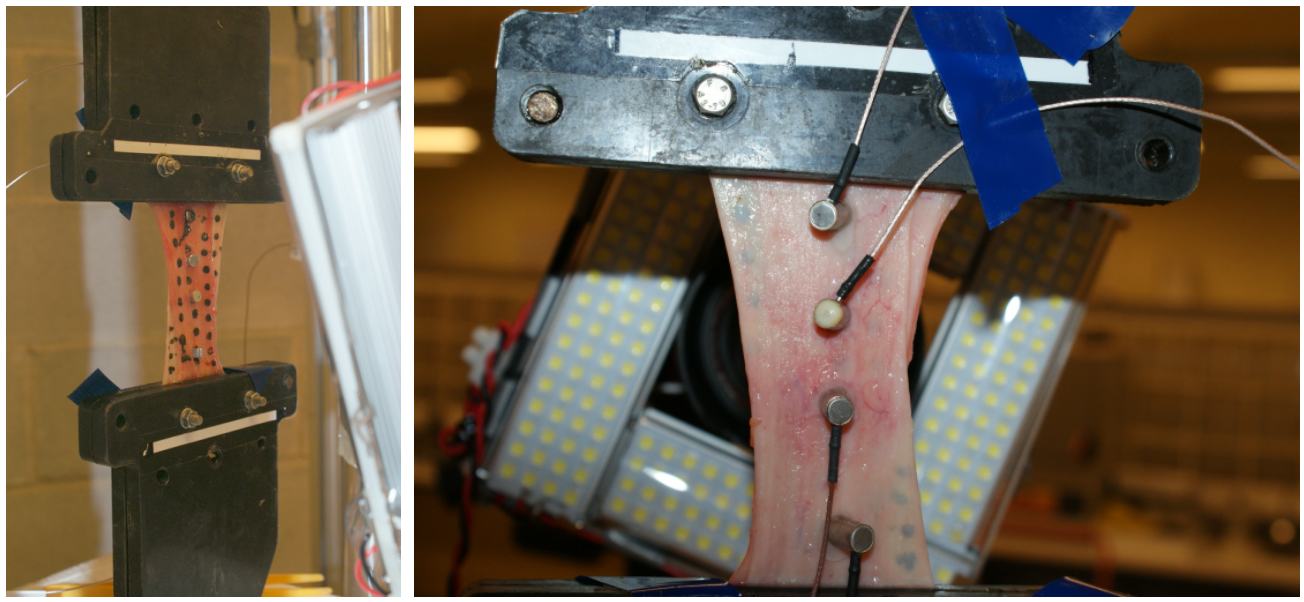

Figure 1: Experimental setting for the uniaxial tensile tests. Right: sample in place for testing, with clamps and acoustic sensors, the upright face is the muscularis externa layer. Left: detail of the acoustic sensors in the close-up face is the mucosa layer.

An important technical issue was the design of the clamps. The clamps 
were made out of non-porous polymeric material (Nylon 6) for two main reasons: (1) a porous material would have produced adherence and local dehydration in the sample, (2) in addition, being less rigid the polymeric material allows a better fit to the soft tissue. As it can be observed in figure 2, the planar clamps are formed by two sets of twin plates, each set is located at the edge of the rectangular sample of tissue. The clamps used for all tests were specifically designed for the occasion. The thickness and dimensions were adjusted in order to ensure that deformations of the clamps are completely negligible and the application of pressures and forces is fairly uniform. Preventing the creeping of the tissue was specially difficult because of the existence of water and moisture in the tissue; which in some cases acted as lubricant. After some preliminary testing and the addition of some extra drills the pressure was increased.

This pressure ensures no significant sliding and, thus, the strain measures are correct (if there had been any sliding, the strain measure would have been distorted). The experimental setting is the same described in [Sánchez-Molina et al., 2014]. For the esophageal tissue, a stress-strain relation of type:

$$
\tau=A e^{-b \varepsilon^{2}} \varepsilon
$$

was obtained the measured parameters were $A=5385 \mathrm{kPa}$ and $b=864.2$ (here $\tau$ is the [Piola] axial stress, and $\varepsilon$ is the [Green-Lagrangian] strain). This relation 1 is deduced from the Yang-Gregersen-Deng constitutive equation [Deng et al.,1994, Yang et al.,2006], and it was found quite accurate $\left(r^{2}=\right.$ 0,9977) for the data. For the purposes of this study, the exact constitutive equation used is not very important, as long as the model approximates well the stress-strain curve (all adequate models lead to a distribution function for the ultimate stress of the same type). The relation (1) is needed to calculate the stress when a failure is detected because the camera measures the strain, not the stress.

In addition, the experimental setup included four acoustic emission sensors (see Fig. 1) that detected the occurrence of micro-failures inside the sample (each micro-failure releases a certain amount of elastic energy which can be detected by sensors). 
(a)

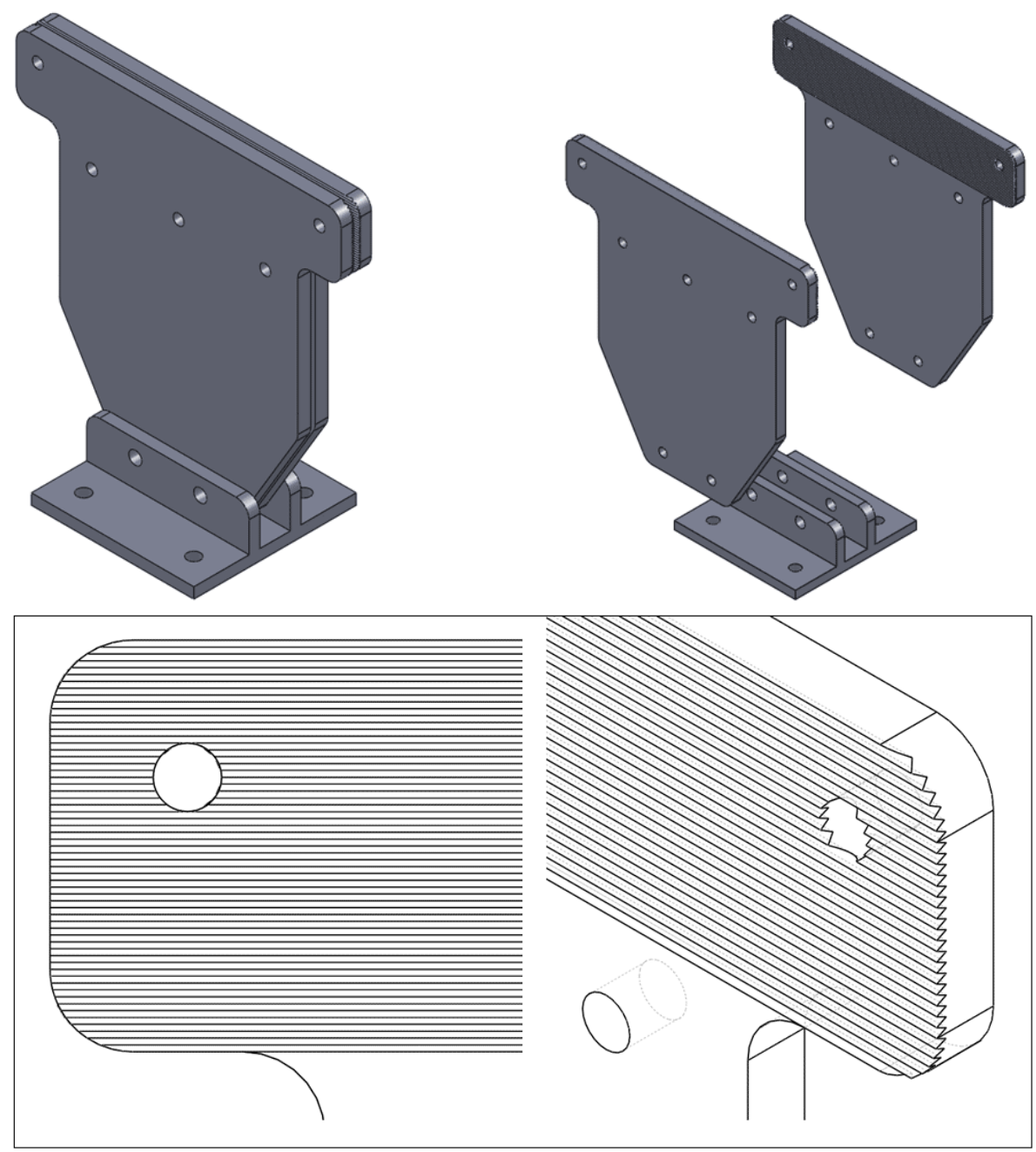

Figure 2: Planar clamps for uniaxial tensile tests: Top (a) the pair of clamps in position for holding the sample, (b) Exploded assembly. Bottom Details of the fluted surface.

\subsection{Acoustic Emission Setup}

The Acoustic Emission (AE) technique is a Non-Destructive Testing (NDT) ${ }_{112}$ method based on stress wave detection. Micro-structural damages or changes ${ }_{113}$ in an elastic material generate elastic waves that can be detected by AE ${ }_{114}$ 
[Scruby, 1987, Drouillard,1996]. This technique has widely been used as an NDT in engineering applications and materials research, including use in biological tissue [Kohn, 1995, Paschos et al., 1984]. AE is a very sensitive passive technique that does not interfere in the test. This makes it a specially useful technique and has been used for predicting failure of different types of materials, including composites and materials whose micro-structure contains fibers [Giordano et al.,1998, Huguet, et al.,2002].

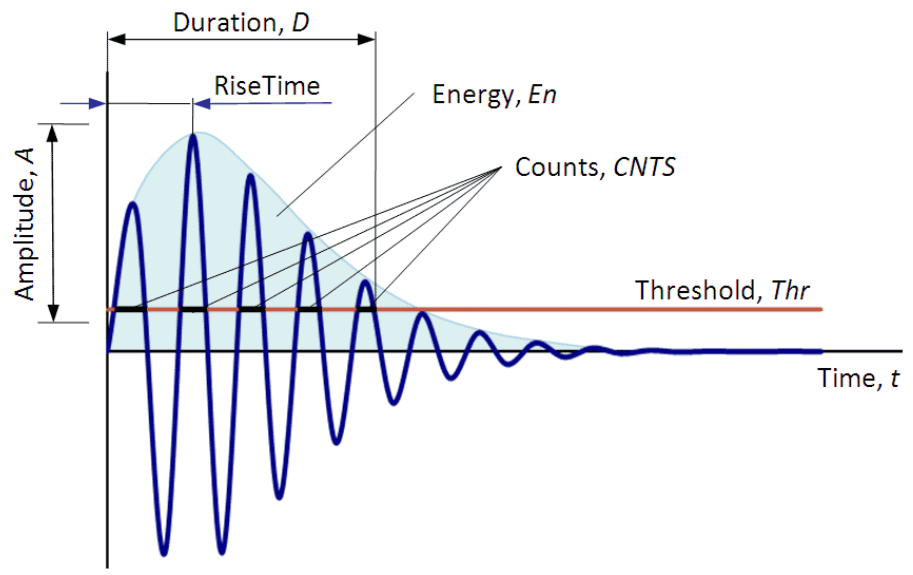

Figure 3: Main features of a stress wave detectable by acoustic emission. The threshold is used to discriminate relevant $\mathrm{AE}$ signals. The duration, the amplitude and the number of counts allow both the calculation of energy and the filtering of signals.

AE signals emitted during the tensile test were detected and recorded with a Vallen System Gmbh. Four miniature AE sensors were magnetically attached to the esophagus specimen (see Fig. 1). The sensors applied in the middle of specimen act as signal sensors while upper and lower sensors act as guard sensors to filter the signals from the grips. All four are of resonant type (VS700D, Vallen System Gmbh) with a frequency range between $100-$ $800 \mathrm{kHz}$ and a peak frequency of $600-800 \mathrm{kHz}$ (flat range). Four $34 \mathrm{~dB}$ preamplifiers (AEP4) and four channel system (AMSY-5) were used. The AE system provides frequency filtering in the pre-amplifiers and on the AMSY-5 board. The filter is a band-pass type, and it was set to $95-850 \mathrm{kHz}$. The AE measurements were filtered using a low threshold of $34 \mathrm{~dB}$, and signals with zero duration and/or zero rise time were removed (the AE signal features are shown in Fig. 3).

In this study, a detected signal whose energy exceeds the energy threshold 
and is almost "punctual in time" is called a micro-failure. It has been checked 136 that visible breaking of fibers produces such a signal. We do not claim ${ }_{137}$ that every signal is the breaking of a single fiber. It could be that some of ${ }_{138}$ the micro-failures detected involve the breaking of groups of fibers. In fact, ${ }_{139}$ we have found that the micro-failures differ significantly in energy as it is ${ }_{140}$ reflected in equation (12).

\subsection{Stochastic models}

The fiber breaking process can be represented by means of two random vari- ${ }^{143}$ ables $\left(N_{\tau}, D_{\tau}\right)$ where $N_{\tau}$ represents the number of micro-failures (or number of "broken" fibers) and $D_{\tau}$ the cumulative damage when the level of stress is given by $\tau$. The number of micro-failures $N_{\tau}$ is a [non-homogeneous] renewal process, i.e. a stochastic process constructed from a simple [nonhomogeneous] Markov process [Whitt,1982]:

1. Let $S_{1}, S_{2}, \ldots, S_{n}$ be a sequence of increases in stress, $S_{k+1}$ represents ${ }_{149}$ the stress increase between $k$ th micro-failure and $(k+1)$-th micro- ${ }_{150}$ failure (we assume the expected values $\mathbb{E}\left(S_{k}\right)$ of the stress increases $S_{k}{ }_{151}$ are finite, $\left.0<\mathbb{E}\left(S_{k}\right)<\infty\right)$.

2. Define for each $n$ :

$$
T_{n}=\sum_{i=1}^{n} S_{i}
$$

then $T_{n}$ is the cumulative stress level at which the $n$th micro-failure ${ }_{154}$ occurs.

3. Finally the variable $N_{\tau}=\sup \left\{n \mid T_{n} \leq \tau\right\}$ the number of micro-failures ${ }_{156}$ up to the stress level $\tau$.

Assume that the $i$ th micro-failure involves a damage $d_{i}$ (in practice, this is ${ }_{158}$ evaluated by the amount of energy released and detected by the AE sensors). ${ }_{159}$ Then the cumulative damage is given by

$$
D_{\tau}=\sum_{k=1}^{N_{\tau}} d_{k}
$$


The $D_{\tau}$ satisfies the conditions for being a [non-homogeneous] "renewalreward process" (here the "reward" at $k$ th step is the damage $d_{k}$ ), alternatively, some authors call this type of stochastic process a "shock process" [Aven \& Jensen,1999, Klefsjö,1981]. In this study, the total damage $D_{\tau}$ is the total dissipated energy recorded by the AE sensors.

Some assumptions about the pair $\left(N_{\tau}, D_{\tau}\right)$ are needed. First we assume that the random variables $S_{k}$ are not identically distributed. The expected values of the variables satisfy:

$$
\mathbb{E}\left[S_{k+1}\right] \leq \mathbb{E}\left[S_{k}\right]
$$

This is so because after some micro-failures have occurred in a tissue there is some internal deterioration and the risk of an additional microfailure increases. In addition, we assume that the stress increase between two successive micro-failure is due the so called "memoryless property":

$$
\mathbb{P}\left(S_{k}>\tau_{2}+\tau_{1} \mid S_{k}>\tau_{1}\right)=\mathbb{P}\left(S_{k}>\tau_{2}\right)
$$

where $\mathbb{P}(\cdot)$ denotes the probability measure. We can satisfy the condition (5) if for each $k$ the random variable $S_{k}$ follow an exponential distribution [Breuer \& Baum,2005]. To deal with the condition (4), we introduced a feature used in the problem of machine maintenance developed in [Yeh,1988], where the time between two successive breakdowns decreases. The idea is to consider a deterioration parameter $a=\exp (\beta)$ [where $\beta>0$ represents the "deterioration rate"] and to assume that "undeteriorated" random variables $\hat{S}_{k}$ :

$$
\hat{S}_{k}:=S_{k} a^{k-1}=e^{\beta(k-1)} S_{k} \sim \operatorname{Exp}(\lambda)
$$

are identically random variables distributed according to an exponential distribution: $\hat{S}_{k} \sim \operatorname{Exp}(\lambda)$ (i.e. all the variables $\hat{S}_{k}$ are distributed according to an exponential distribution of parameter $0<\lambda<\infty$ ). Thus, in this study, the occurrence of micro-failures in time is modeled by a stochastic process with two parameters: $\lambda$ and $\beta$. The exponential factor in (6) ensures a series of successive micro-failures with increasingly shorter "times" (stress increases) between micro-failures. These assumptions are qualitatively suggested by the observed behavior.

For the distribution function of the damage $d_{k}$ associated with the $k$ th micro-failure, the empirical distribution function is used. Therefore, the proposed model of failure is a stochastic process of type: 


$$
\left\{\left(N_{\tau}, D_{\tau}, \mathcal{F}_{\tau}\right) \mid \tau \in \mathbb{R}^{+},\left(N_{\tau}, D_{\tau}\right) \in \mathbb{R}^{2}\right\}
$$

where $\tau=\tau\left(\sigma_{i j}\right)$ is the "equivalent stress" (i.e. a scalar function of the ${ }_{192}$ component of the stress tensor $\left.\sigma_{i j}\right), N_{\tau}$ as defined above and $D_{\tau}$ given by ${ }_{193}$ (3). In the present case we used $\tau=$ the first principal stress. Formally, for ${ }_{194}$ each $\tau$ the triplet $\left(N_{\tau}, D_{\tau} ; \mathcal{F}_{\tau}\right)$ is a random variable on $\mathbb{R}^{2}$ (here, $\mathcal{F}_{\tau}$ is the ${ }_{195}$ associated $\sigma$-algebra). $\quad 196$

The experimental data are used to estimate the parameters of the model ${ }_{197}$ by comparing the current stress $\tau$ and the previous damage (the number ${ }_{198}$

of micro-failures $N_{\tau}$ and the cumulative damage $D_{\tau}$ ). Obviously, as the ${ }_{199}$ stress and the cumulative damage increase, the stress span between any two 200 consecutive micro-failures diminishes. The increasing probability per unit ${ }_{201}$ of time implies that we have a non-homogeneous continuous-time stochastic 202 process. Note that in this model no hypothesis about the nature of fiber ${ }_{203}$ breaking is done.

\subsection{Parameter Estimation}

The proposed stochastic model has two defining parameters $\beta$ and $\lambda$. The ${ }_{206}$ best fitting for these two parameters is computed by minimizing a penalty ${ }_{207}$ function $\phi(\beta, \lambda)$. The penalty function is a sum of squares. This penalty ${ }_{208}$ function has a minimum and the minimum is achieved for the best-fitting ${ }_{209}$ parameters. The procedure for finding the minimum of the penalty function 210 is indeed a least-squares non-linear regression. The penalty function is given ${ }_{211}$ by the differences between expected values and sample values:

$$
\phi(\beta, \lambda)=\left[\mu_{\infty}(\beta, \lambda)-\bar{\sigma}_{u}\right]^{2}+\sum_{i=1}^{n}\left[\mu_{i, i+1}(\beta, \lambda)-\left(\sigma_{i+1}-\sigma_{i}\right)\right]^{2}
$$

where $\mu_{i, i+1}$ is the expected value of the stress increase between the $i-{ }_{213}$ th failure and the $(i+1)$-th failure, the $\sigma_{i}$ are the corresponding actual ${ }_{214}$ experimental stresses. And $n=470$ is given by the number of stress increasses 215 between microfailures. Additionally, $\mu_{\infty}$ is the expected value of the stress 216 increase between the first detected micro-failure and the ultimate stress; and ${ }_{217}$ $\bar{\sigma}_{u}$ is the corresponding observed increase.

The expected values $\mu_{\infty}$ and $\mu_{i, j}$ can be derived from (2)-(6). A com- ${ }_{219}$ putation of the expected values is presented in the Appendix [see equations ${ }_{220}$ (17) and (19)]: 


$$
\left\{\begin{array}{l}
\mu_{\infty}=\frac{1}{\left(1-e^{-\beta}\right) \lambda} \\
\mu_{i, j}=\frac{e^{-\beta(i-1)}-e^{-\beta j}}{\left(1-e^{-\beta}\right) \lambda}
\end{array}\right.
$$

The best-fitting parameters were obtained by solving the system of nonlinear equations:

$$
\left\{\begin{array}{l}
\frac{\partial \phi}{\partial \beta}=0 \Rightarrow \quad\left[\mu_{\infty}-\bar{\sigma}_{u}\right] \frac{\partial \mu_{\infty}}{\partial \beta}+\sum_{i=1}^{n}\left[\mu_{i, i+1}-\sigma_{i+1}+\sigma_{i}\right] \frac{\partial \mu_{i, i+1}}{\partial \beta}=0 \\
\frac{\partial \phi}{\partial \lambda}=0 \Rightarrow \quad\left[\mu_{\infty}-\bar{\sigma}_{u}\right] \frac{\partial \mu_{\infty}}{\partial \lambda}+\sum_{k=1}^{n}\left[\mu_{i, i+1}-\sigma_{i+1}+\sigma_{i}\right] \frac{\partial \mu_{i, i+1}}{\partial \lambda}=0
\end{array}\right.
$$

\section{Results}

The solution of the nonlinear system 10 provides an estimation of the parameters $\lambda$ and $\beta$. For solving it a Mapple 17 script was used. The procedure involves estimate initial values for $\lambda_{0} \approx \lambda$ and $e^{-\beta_{0}} \approx e^{-\beta}$ and then a NewtonRaphson procedure was applied to compute $\lambda$ and $\beta$. For both pre-estimation the equation (17) was used putting $\mu_{\infty}=t_{\infty}$, thus for the initial values $\lambda_{0}$ and $\beta_{0}$ :

$$
\lambda_{0}:=\frac{t_{\infty}}{1-e^{-\beta_{0}}}
$$

Then equation $\partial \phi\left(\lambda_{0}, \beta_{0}\right) / \partial \beta=0$ is solved and a value $\beta_{0}$ is computed this also provides a value for $\lambda_{0}$ according to (11). These values were used to find solutions for $\beta \in[0,100]$ and $\lambda \in\left(0,10 \lambda_{0}\right]$. This procedure had been tested for computed data with previously known values, and had proven to be robust for all the range of values.

The computed parameters for the sample of this study are $\beta=0.0774$ and $\lambda=0.0196\left[\mathrm{kPa}^{-1}\right]$.

\subsection{Number of micro-failures}

The AE equipment provides a list of detected micro-failures or "hits" versus time. We can plot the number micro-failure versus measured stress (see Fig. $4)$. 


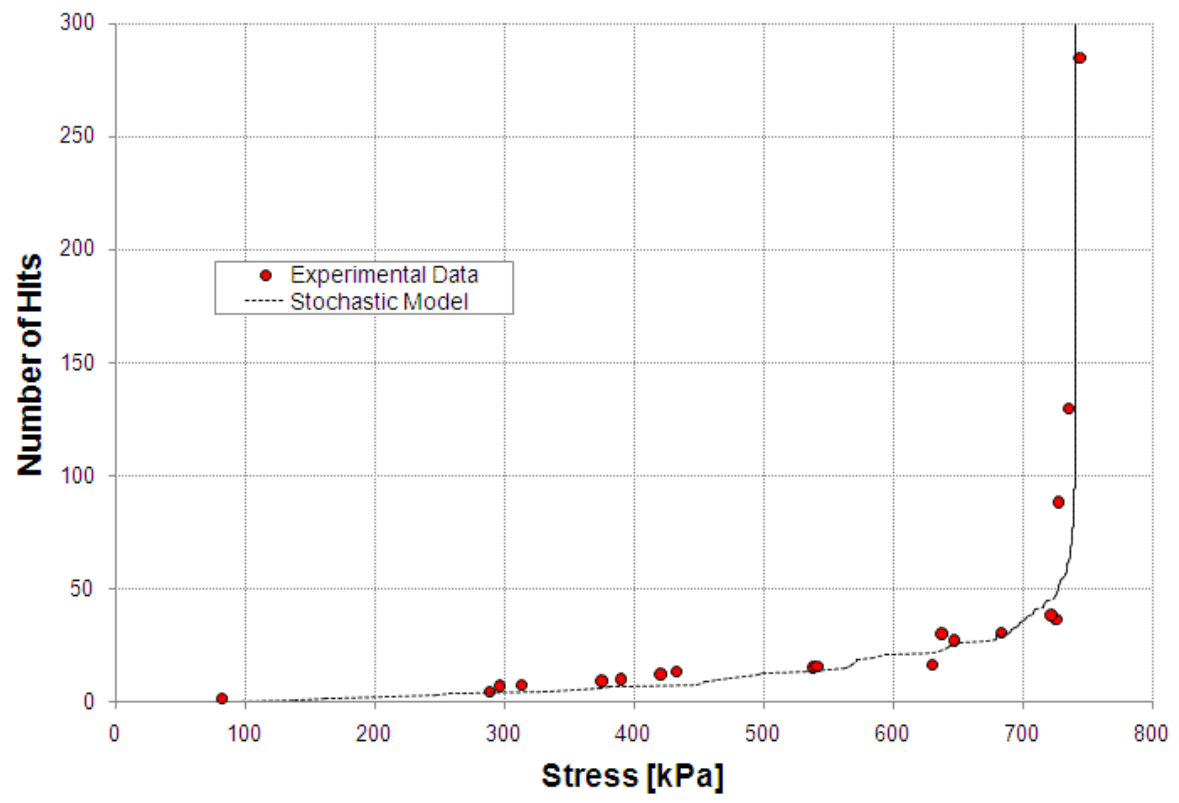

Figure 4: The number of micro-failures or hits versus the strain level, for a stress $\tau<100$ $\mathrm{kPa}$ no micro-failures are observed (red spots). From this threshold, we observe some hits, and around $\tau \approx 720 \mathrm{kPa}$ an exponential increase in the number of micro-failures is observed. The dotted line correspond to a simulated curve using the computed parameters $\lambda, \beta$. The observed data and the simulated curve show high resemblance.

In figure 4 , we see that a chain of successive micro-failures begins for ${ }_{242}$ $\tau>100 \mathrm{kPa}$, and then, the rate of micro-failures accelerates abruptly for ${ }^{243}$ $\tau \approx 720 \mathrm{kPa}$, when the number of hits increases exponentially. Then, the ${ }_{244}$ cumulative effect of all these micro-failures around $\tau \approx 720 \mathrm{kPa}$ implies the ${ }_{245}$ rapid deterioration of the tissue and the final macroscopic failure. Thus in ${ }_{246}$ this model, the macroscopic failure is interpreted as a situation when a huge ${ }_{247}$ number of micro-failures are produced for a specific value of stress. The ${ }_{248}$ graphic for the number of micro-failures presents a vertical asymptote for 249 the value at which macroscopic failure occurs. Namely, the ultimate stress if 250 of the form $\sigma_{u}=\sigma_{0}+T_{\infty}$ (where $\sigma_{0}$ is the threshold stress below which there ${ }^{251}$ is no fiber-breaking, and $T_{\infty}$ is the limit random variable given by (2) when ${ }_{252}$ $n \rightarrow \infty)$. This kind of catastrophic behavior is also found in other type of ${ }_{253}$ probabilistic models in the literature [Pradhan, \& Hansen, 2005], although ${ }^{254}$ the probabilistic basis of these other models is different.

The next section shows that the "damage" or dissipated energy attains ${ }_{256}$ 
an arbitrarily high value when the number of micro-failures increases exponentially. Thus, after some prefixed level of damage, the tissue finally breaks down.

\subsection{Distribution of dissipated energy}

The same tendency can be seen for energy or damage (see Fig. 5). This figure shows the elastic energy lost in form of broken fibers. Around $\sigma \approx 720$ $\mathrm{kPa}$. The tissue is unable to resist such an increase of damage and all the energy supplied by the test machine is occupied in breaking fibers. This finally causes the complete failure of the tissue.

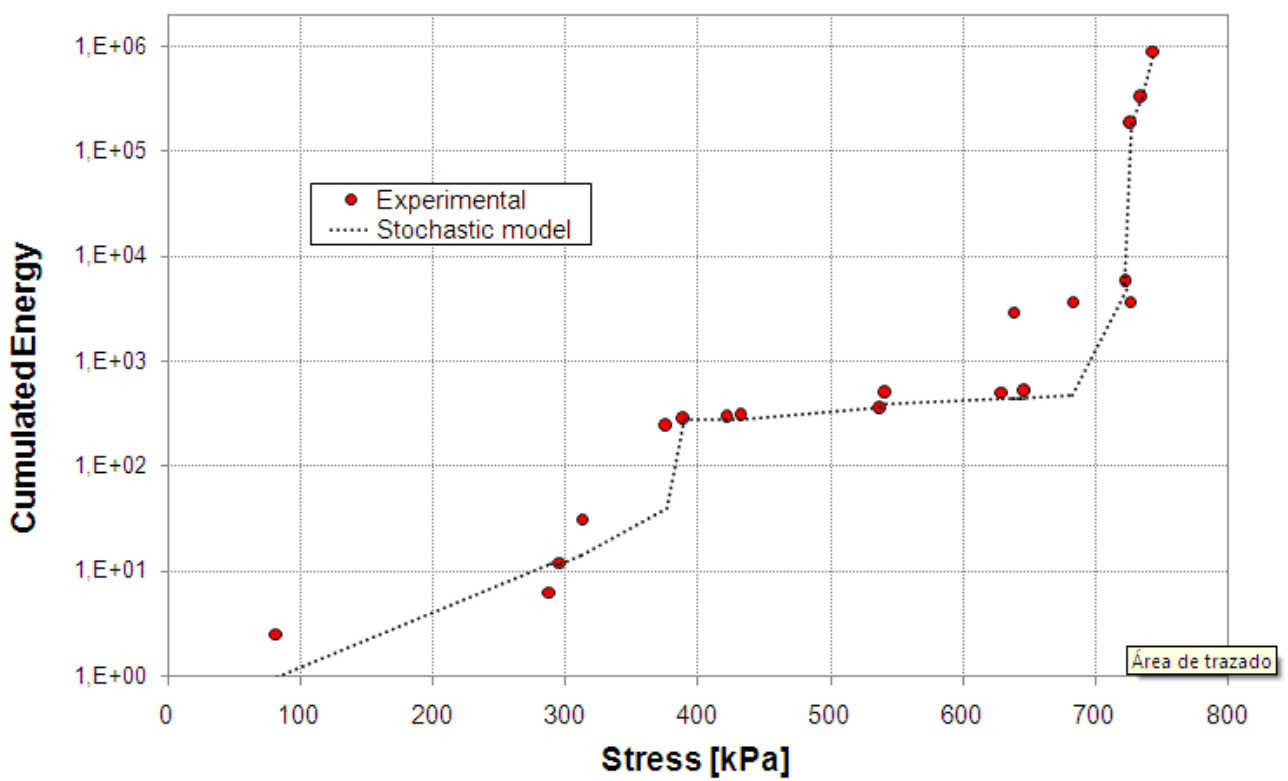

Figure 5: The dissipated energy in inelastic failures or breaking of fibers: red dots are the measured energies, and the dashed line is a simulated curve with the same parameters than the measured data.

In order to verify the accuracy of the model, the distribution of the damage per micro-failure $E$, i.e. the energy dissipated for each hit detected was investigated. As in other critical phenomena involving a high number of elements, the expected distribution of $E$ was a power law or Pareto distribution (because, $\log (E)$ is exponentially distributed, see Fig. 6). The power law is a scale-free distribution. This kind of distributions is found in phase transi- 


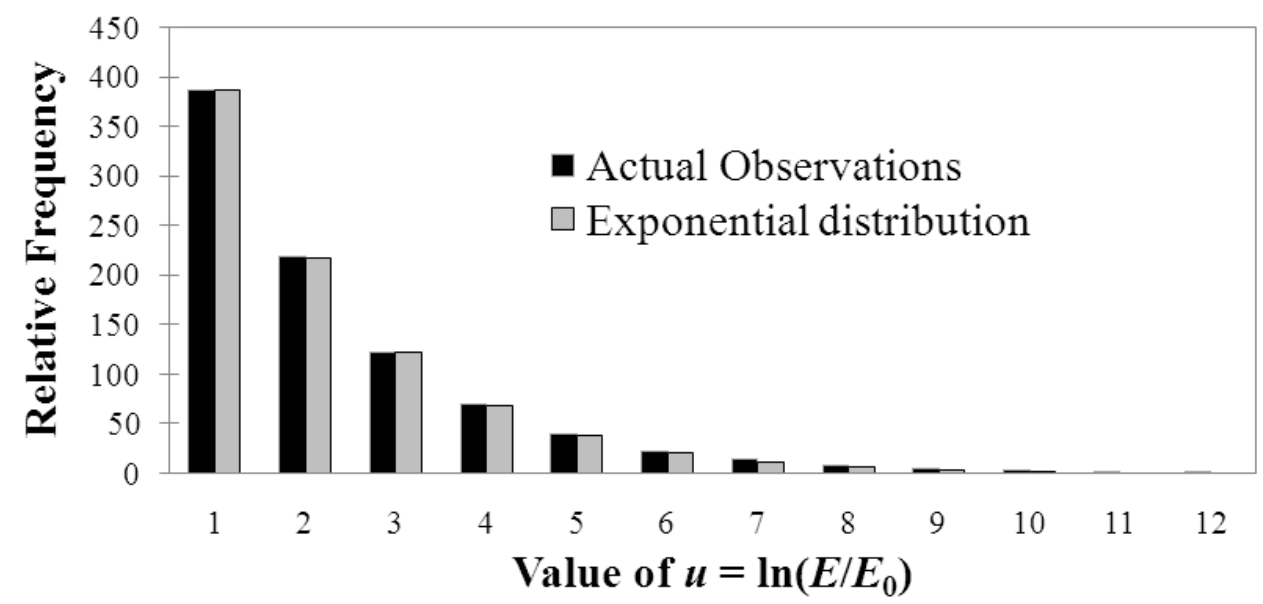

Figure 6: Histogram of the logarithm dissipated energy per micro-failure $(u)$, compared to an exponential distribution with parameter $\alpha=1.198$

tions [Uchino \& Nomura,1982, Panagiotou et al., 1984], economical crashes ${ }^{272}$ [Laloux et al.,2009], avalanches and other critically self-organized phenom- ${ }^{273}$ ena [Bak et al.,1988]. Other probabilistic models predicts the occurrence of ${ }^{274}$ avalanches of fiber failure [Pradhan, \& Hansen, 2005] (but formal details of ${ }_{275}$ these models are different from the stochastic one presented in this paper). ${ }^{276}$ An accurate account of the data showed that the energies of micro-failures 277 follow effectively a Pareto distribution of the form:

$$
f_{E}(E)=\frac{\alpha}{E_{0}}\left(\frac{E_{0}}{E}\right)^{1+\alpha}
$$

where $\alpha$ is a decay parameter and $E_{0}$ is an arbitrary constant depending ${ }_{279}$ on the units of energy used. Making the change $u=\ln \left(E / E_{0}\right)$, the equation 280 (12) implies that the logarithm of the energy per micro-failure $u$ is distributed ${ }_{281}$ according to an exponential law:

$$
f_{u}(u)=\alpha \mathrm{e}^{-\alpha u}
$$

This latter form is more convenient for finding the decay parameter $\alpha$. ${ }^{283}$ The Fig. 6 shows a comparison the histogram for the actual observations of 284 energy per micro-failure and the best-fitting exponential distribution for the 285 observations. The fitting of the exponential distribution to the observations 286 is very good (Kolmogorov-Smirnov test, and $\chi^{2}$ test: $p>0.9999$ ) and the ${ }_{287}$ computed parameter results to be $\alpha=1.198$. This means that energy per ${ }_{288}$ 
micro-failure satisfies $\mathbb{P}(E>w) \propto w^{-1.198}$. As we pointed out in the section 2.1 , this latter distribution refers to micro-failures and it is possible that some micro-failures involve groups of fibers.

\subsection{Distribution of predicted maximum stress}

It is interesting to note that even for given values of the model $(\beta, \lambda)$, the maximum stress $\left(\sigma_{u}\right)$ resisted by the tissue is not a fixed value, but it lies within a range because it is a stochastic model. So, it makes sense to look at the distribution of predicted maximum stress. A great number of simulations have been done $(n=500)$ in order to generate the distribution of $\sigma_{u}$. We define previously, $\bar{\sigma}_{u}=\sigma_{u}-\sigma_{0}$ where $\sigma_{0}$ is the stress level for which the microfailures begin. The simulations used a Montecarlo method for estimating a great number of values for variables of type $\hat{S}_{k}$ that are distributed according to an exponential distribution [see equation (6)], the variables $S_{k}=e^{-\beta(k-1)} \hat{S}_{k}$ were computed and the value of $T_{\infty}$ is computed. By repeating this process $(n=500)$, a good sample for $\bar{\sigma}_{u}$ is obtained.
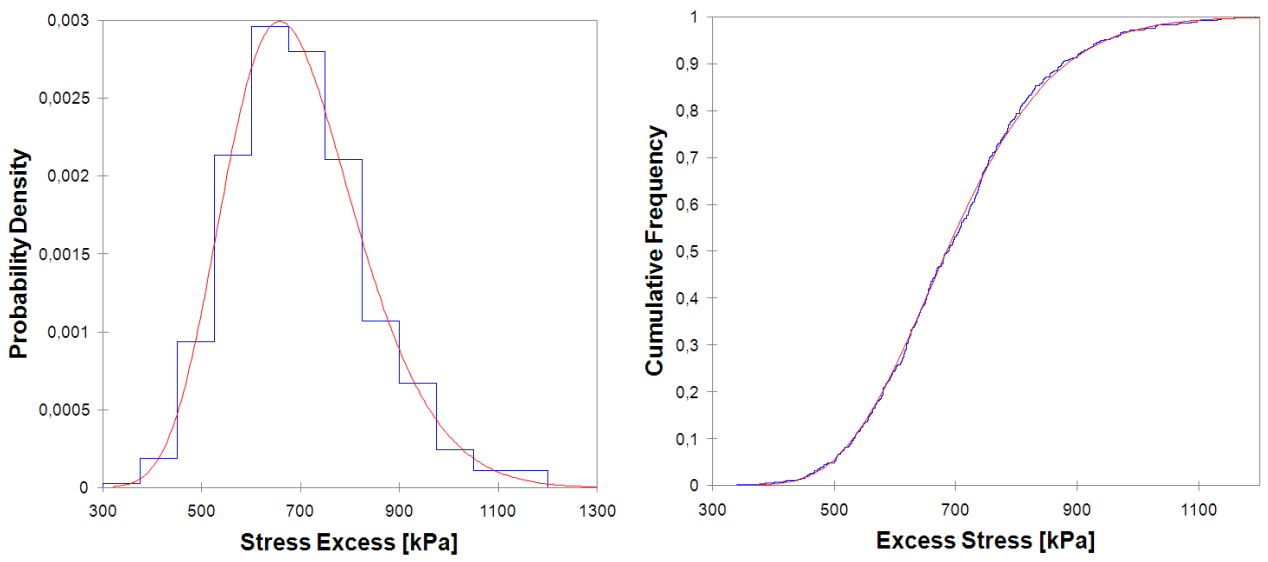

Figure 7: Empirical distribution for stress $\bar{\sigma}_{u}$ and adjusted model.

The empirical distribution (see Fig. 7) of $\bar{\sigma}_{u}$ seems to approximate pretty well to a Generalized Extreme Value distribution (GEV distribution) of Fréchet type, with cumulative distribution function:

$$
F\left(\bar{\sigma}_{u} ; \bar{\mu}, k, \xi\right)=\exp \left\{-\left[1+\xi\left(\frac{\bar{\sigma}_{u}-\bar{\mu}}{k}\right)\right]^{-1 / \xi}\right\}
$$


The empirical parameters obtained from simulated data were $\xi=0.146, k=307$ $124.3 \mathrm{kPa}$ and $\bar{\mu}=637.2 \mathrm{kPa}$ (Kolmogorov-Smirnov tests gives $p>0.9944$ ). 308 These values were estimated with a standard statistical package. Note that 309 expected value is given by:

$$
\begin{aligned}
& \mathbb{E}\left(\bar{\sigma}_{u}\right)=\bar{\mu}+\frac{k}{\xi}-\frac{k}{\xi} \Gamma(1+\xi) \approx 693.1 \\
& \mathrm{SD}\left(\bar{\sigma}_{u}\right)=\frac{k}{\xi} \sqrt{\Gamma(1+2 \xi)-\Gamma^{2}(1+\xi)} \approx 136.2
\end{aligned}
$$

This is consistent with the expected value of the random variable $T_{\infty}\left[={ }_{311}\right.$ $\left.\lim _{n} T_{n}\right]$. According to the Appendix we have:

$$
\begin{aligned}
& \mathbb{E}\left(T_{\infty}\right)=\frac{1}{\left(1-e^{-\beta}\right) \lambda}=693.62 \\
& \operatorname{SD}\left(T_{\infty}\right)=\frac{1}{\lambda} \frac{e^{\beta}}{\sqrt{e^{2 \beta}-1}}=135.18
\end{aligned}
$$

The explicit derivation of the form of the equation (14) is discussed in the ${ }_{313}$ appendix, but the computations are not simple. For this reason we relied on 314 numerical simulations.

\section{Discussion}

The model presented in this study can explain why the strength stress for 317 a soft tissue material is not necessarily a fixed value, but a random variable ${ }_{318}$ with well defined distribution. The actual strength value obtained in one 319 experiment will depend on very fine accidents in the intertwined fibers. In 320 each experiment, the micro-structure is microscopically and highly random, 321 for this reason not all the similar specimens present the same strength value. $\quad 322$ The best we can have is most likely a method for estimating the true distri- ${ }_{323}$ bution of strength, which is the main contribution of this article.

The idea of representing the mechanical failure by means of a proba- 326 bility density function is not new, however the way in which such a den- ${ }^{327}$ sity is introduced in this article is innovative. Other authors have tried to 328 explain the failure of materials (specially brittle and quasi-brittle ceramic ${ }_{329}$ materials) using probabilities, mainly, the Extreme Value theory (EV the- ${ }_{330}$ ory), and the Weibull distribution. Weibull distribution is a kind of Extreme ${ }_{331}$ 
Value distribution. Many studies assume that at small scales the strength of the material is given by a distribution related to Weibull distribution [Sutcu,1989, Manzato et al.,2009]. Unlike these models, we do not assume special distributions at small scales instead we used a probabilistic machinery based on stochastic processes (and not directly on EV theory). The type of stochastic treatment used here allows to compute the parameters $\lambda$ and $\beta$ characterizing the original arrangement of fibers, and therefore, we can compute from them the distribution of ultimate stress by simulation. We found that the distribution of the strength of soft tissues is a Fréchet distribution, another type of Extreme Value distribution (indeed, Fréchet, Weibull and Gumbel distributions are the only three possible types of EV distributions and together they form the family of Generalized Extreme Value distributions).

However, it is possible that the GEV distributions are not the only type of distributions relevant for strength of materials. For example, an influent work in percolation models found a completely new type of distribution for the strength of the material [Duxbury et al.,1987]. Further work is needed to clarify how many the distributions are possible, our study shows that Fréchet distribution is one of the relevant distributions has to be considered.

Another interesting finding of this study is that fiber breaking in soft tissues present distributions of the same type found in other critical phenomena associated with a great number of elements in interaction, namely the presence of power-law or Pareto distributions. Further work is needed in order to interpret the decay parameter $\alpha$, but it is interesting to note the presence of such an exponent in the distribution function of "damage".

About figures 4 and 5, it is important to highlight that we are dealing with stochastical, not deterministic processes. Thus, a simulation consists in a random sequence of microfailures, so the red dots represent the sequence of real microfailures in the tissue, and the dashed line is a simulated stochastical (random) curve using the same parameters computed for the sequence of real microfailures. In figure 4, both the simulated process and the observed process fit very well. In figure 5 , both processes are qualitatively similar (but remember that red dots and the dashed line depict different instances of random processes with the same statistical parameters, so the dashed line is not an adjusted curve but only a qualitatively similar curve).

Finally, note that in this study, all the statistics are referred to the set 
of data of one specimen. The number of micro-failures $\left(n_{m f}=285\right)$ supplied 370 by this specimen seems to be sufficient in order to achieve significance in 371 various statistical tests. In addition, the adjusted parameters are adequate to 372 generate a great number of different simulations $\left(n_{\text {sim }}=500\right)$. But it would 373 be interesting to have different instrumented specimens in order to make 374 inter-specimen statistics comparing the obtained parameters. Unfortunately, 375 the current data does not allow such a comparison. A further step would be ${ }_{376}$ to obtain such data.

\section{Appendix: Mean and Variance of $T_{\infty}$}

In this appendix we calculate the expected values and variances of the vari- 379 ables needed for the comparison with the experimental data and the fitting 380 of the parameters in equation (8).

First, we consider the variable $T_{\infty}=\lim _{n} T_{n}$, where the variables $T_{n}$ were 382 defined in equation (2). The mean and the variance of this variable can be ${ }_{383}$ computed directly. For the mean $\mu=\mathbb{E}\left(T_{\infty}\right)$ :

$$
\mu_{\infty}=\mathbb{E}\left(T_{\infty}\right)=\mathbb{E}\left(\sum_{k=1}^{\infty} e^{-\beta(k-1)} \tilde{S}_{k}\right)=\sum_{k=1}^{\infty} e^{-\beta(k-1)} \mathbb{E}\left(\tilde{S}_{k}\right)=\frac{1}{\left(1-e^{-\beta}\right) \lambda}
$$

Second, for the computation of $\sigma_{\infty}^{2}=\operatorname{Var}\left(T_{\infty}\right)$, we use $\sigma_{\infty}^{2}=\mathbb{E}\left(T_{\infty}^{2}\right)-{ }_{385}$ $\mathbb{E}^{2}\left(T_{\infty}\right)$. We compute previously $T_{\infty}^{2}$ :

$$
\begin{aligned}
T_{\infty}^{2} & =\left(\sum_{k=1}^{\infty} e^{-\beta(k-1)} \tilde{S}_{k}\right)^{2}= \\
& =\sum_{k=1}^{\infty} \sum_{l=1}^{k}\left(\tilde{S}_{l} e^{-\beta(l-1)}\right)\left(\tilde{S}_{k+1-l} e^{-\beta((l+1-l)-1)}\right) \\
& =\sum_{k=1}^{\infty} e^{-\beta(k-1)} \sum_{l=1}^{k} \tilde{S}_{l} \tilde{S}_{k-l+1}
\end{aligned}
$$

Taking now expected values:

$$
\begin{aligned}
\mathbb{E}\left(T_{\infty}^{2}\right) & =\sum_{k=1}^{\infty} e^{-\beta(k-1)} \sum_{l=1}^{k} \mathbb{E}\left(\tilde{S}_{l} \tilde{S}_{k-l+1}\right)= \\
& =\sum_{k=1}^{\infty} \frac{e^{-\beta(k-1)}}{\lambda^{2}}\left(k+\frac{1+(-1)^{k+1}}{2}\right) \\
& =\frac{1}{\lambda^{2}\left(1-e^{-\beta}\right)^{2}}+\frac{1}{\lambda^{2}\left(1-e^{-2 \beta}\right)}
\end{aligned}
$$

And, finally, we obtain using (17): 


$$
\sigma_{\infty}^{2}=\mathbb{E}\left(T_{\infty}^{2}\right)-\mathbb{E}^{2}\left(T_{\infty}\right)=\frac{1}{\lambda^{2}} \frac{e^{2 \beta}}{e^{2 \beta}-1}
$$

More in general, the random variables $T_{i, j}$ used for the fitting of parameters have expected values given by:

$$
\begin{aligned}
\mu_{i, j}=\mathbb{E}\left(T_{i, j}\right) & =\mathbb{E}\left(\sum_{k=i}^{j} e^{-\beta(k-1)} \tilde{S}_{k}\right) \\
& =\sum_{k=i}^{j} e^{-\beta(k-1)} \mathbb{E}\left(\tilde{S}_{k}\right)=\frac{e^{-\beta(i-1)}-e^{-\beta j}}{\left(1-e^{-\beta}\right) \lambda}
\end{aligned}
$$

The above calculations are sufficient for adjusting parameters and showing the accuracy of the model. Another interesting question is to obtain directly the probability density distribution of $T_{\infty}$. Technically, the probability distribution for the ultimate stress is computable but it involves infinite convolution products. In particular, some results of [Kawata, T. \& Udagawa, M.,1949] can be used to obtain the characteristic function of the distribution of $T_{\infty}$ which is the infinite product:

$$
\varphi_{T_{\infty}}(s)=\mathbb{E}\left[e^{i s T_{\infty}}\right]=\prod_{i=1}^{\infty}\left(1-\frac{i s}{\lambda e^{-\beta(k-1)}}\right)^{-1}
$$

The computation necessary to write explicitly the probability function of $T_{\infty}$ from the above expression is complex. For this reason, we have preferred numerical simulations to obtain the empirical distribution.

\section{References}

[Aven \& Jensen,1999] Aven, T. \& Jensen, U.: Stochastic models in reliability (book), ed. Springer, 1999.

[Bak et al.,1988] Bak, P., Tang, Ch., Wiesenfeld, K.: "Self-organized criticality", Physical review A, 38(1), p. 364, 1988.

[Breuer \& Baum,2005] Breuer, L. \& Baum, D.: An introduction to queueing theory and matrix-analytic methods (book), ed. Springer, 2005.

[Deng et al.,1994] Deng, S.X., Tomioka, J., Debes, J.C., Fung, Y.C.: "New experiments on shear modulus of elasticity of arteries", American Jour- 
nal of Physiology-Heart and Circulatory Physiology, 35(1), pp. 1-10, ${ }_{411}$ 1994.

[Drouillard,1996] Drouillard, T.F.: "A history of acoustic emission", Journal ${ }_{413}$ of Acoustic Emission, 14(1), pp. 1-34, 1996.

[Duxbury et al.,1987] Duxbury, P.M., Leath, P.L., Beale, P.D.: "Breakdown ${ }_{415}$ properties of quenched random systems: The random-fuse network", 416 Physical Review B, 36(1), p. 367, 1987.

[Giordano et al.,1998] Giordano, M., Calabro, A., Esposito, C., D'amore, A., ${ }_{418}$ Nicolais, L.: "An acoustic-emission characterization of the failure modes ${ }_{419}$ in polymer-composite materials", Composites Science and Technology, 420 58(12), pp. 1923-1928, 1998.

421

[Gugala \& Lindsey,2003] Gugala, Z., and Lindsey, R. W.: "Classification of ${ }_{422}$ Gunshot Injuries in Civilians", Clin. Orthop. Relat. Res., 1, pp. 65-81, ${ }_{423}$ 2003.

[Huguet, et al.,2002] Huguet, S., Godin, N., Gaertner, R., Salmon, L., Vil- ${ }^{425}$ lard, D.: "Use of acoustic emission to identify damage modes in glass ${ }_{426}$ fibre reinforced polyester", Composites Science and Technology, 62(10), ${ }_{427}$ pp. 1433-1444, 2002.

[Ionescu et al.,2006] Ionescu, I., Guilkey, J. E., Berzins, M., Kirby, R. M. and ${ }_{429}$ Weis, J. A.: "Simulation of soft tissue failure using the material point ${ }_{430}$ method", Journal of biomechanical engineering, 128(6), pp. 917-924, 431 2006.

[Kabir et al.,2006] Kabir, M.R., Lutz, W., Zhu, K., Schmauder, S.: "Fatigue ${ }^{433}$ modeling of short fiber reinforced composites with ductile matrix under ${ }_{434}^{43}$ cyclic loading", Computational materials science, 36(4), pp. 361-366, ${ }_{435}$ 2006 .

[Kawata, T. \& Udagawa, M.,1949] Kawata, T. \& Udagawa, M.: "On infinite ${ }^{437}$ convolutions", Kodai Mathematical Seminar Reports, 1, pp. 15-22, 1949. ${ }_{438}$

[Klefsjö,1981] Klefsjö, Bengt.: "HNBUE survival under some shock models", 439 Scandinavian Journal of Statistics, pp. 39-47, 1981.

440 
[Kohn, 1995] Kohn, D. H.: "Acoustic Emission and Nondestructive Evaluation of Biomaterials and Tissues", Critical ReviewersTM in Biomedical Engineering, 22(3/4), pp. 221-306, 1995.

[Kroon \& Holzapfel, 2008] Kroon and Holzapfel: "A new constitutive model for multi-layered collagenous tissues", Journal of biomechanics, 41(12), pp. 2766-2771, 2008.

[Laloux et al.,2009] Laloux, L., Potters, M., Cont, R., Aguilar, J.-P., Bouchaud, J.-P.: "Are financial crashes predictable?", Europhysics Letters, 45(1), 1999.

[Manzato et al.,2009] Manzato, C., Shekhawat, A., Nukala, P., Alava, M.J., Sethna, J.P., Zapperi, S.: "Fracture strength of disordered media: Universality, interactions, and tail asymptotics", Physical review letters, 108(6), pp. 065504, 2012.

[Natali et al., 2009] Natali, A. N., Carniel, E. L., and Gregersen, H.: "Biomechanical behaviour of oesophageal tissues: material and structural configuration, experimental data and constitutive analysis", Medical engineering $\mathscr{G}$ physics, 31(9), pp. 1056-1062, 2009.

[Panagiotou et al., 1984] Panagiotou, A.D., Curtin, M.W., Toki, H., Scott, D.K., Siemens, P.J.: "Experimental evidence for a liquid-gas phase transition in nuclear systems", Physical Review Letters, 52(7), p. 496, 1984.

[Paschos et al., 1984] Paschos, N.K., Aggelis, D.G., Barkoula, N.-M., Paipetis, A., Gartzonikas, D., Matikas, T.E.,Georgoulis, A.D.: "An Acoustic Emission Study for Monitoring Anterior Cruciate Ligament Failure Under Tension", Physical Review Letters, 53(5), pp. 767-774, 2013.

[Hao et al., 1984] Hao, D.-P., Tang, G., Xia, H., Xun, Zh.-P., Han, K.: "Avalanche process of the fiber-bundle model with stick-slip dynamics and a variable Young modulus", Physical Review E, 87(4), pp. 042126, 2013.

[Pradhan \& Chakrabarti, 2003] Pradhan, S. and Chakrabarti, B. K.: "Failure properties of fiber bundle models", International Journal of Modern Physics B, 17(29), pp. 5565-5581, 2003. 
[Pradhan, \& Hansen, 2005] Pradhan, S. \& Hansen, A.: "Failure properties 474 of loaded fiber bundles having a lower cutoff in fiber threshold distribu- ${ }_{475}$ tion", Physical Review E, 72(2), pp. 026111, 2005.

[Sánchez-Molina et al., 2014] Sánchez-Molina, D., Velázquez-Ameijide, J., 477 Arregui-Dalmases, C., Rodríguez, D., Quintana, V., Shafieian, M. Cran- ${ }_{478}$ dall, J.R.: "A Microcontinuum Model for Mechanical Properties of 479 Esophageal Tissue: Experimental Methodology and Constitutive Anal- ${ }_{480}$ ysis", Annals of biomedical engineering, 42(1), pp. 62-72, 2014.

[Fernández-Esparrach et al., 2011] Fernández-Esparrach, G.,Córdova, H., ${ }_{482}$ Bordas, J. M., Gómez-Molins, I., Ginès, À., Pellisé, M., Sendino, O., ${ }_{483}$ González-Suárez, B., Cárdenas, Á., Balderramo, D.: "Manejo en- ${ }^{484}$ doscópico de las complicaciones de la cirugía bariátrica. Experiencia tras $\quad 485$ más de 400 intervenciones", Gastroenterología y Hepatología, 34(3), pp. ${ }_{486}$ 131-136; 2011, doi: 10.1016/j.gastrohep.2010.12.003

[Scruby, 1987] Scruby, C.B.: "An introduction to acoustic emission", Jour- ${ }_{488}$ nal of Physics E: Scientific Instruments, 20(8), pp. 946-953; 1987, doi: ${ }_{489}$ 10.1088/0022-3735/20/8/001. 490

[Sutcu,1989] Sutcu, M.: "Weibull statistics applied to fiber failure in ceramic ${ }_{491}$ composites and work of fracture", Acta Metallurgica, 37(2), pp. 651-661, 492 1989.

[Uchino \& Nomura,1982] Uchino, K., Nomura, Sh.: "Critical exponents 494 of the dielectric constants in diffused-phase-transition crystals", Fer- ${ }_{495}$ roelectrics, 44(1), pp. 55-61, 1982.

[Whitt,1982] Whitt, W.: "Approximating a point process by a renewal pro- ${ }_{497}$ cess, I: Two basic methods", Operations Research, 30(1), 1982.

[Yeh,1988] Yeh, Lam: "A note on the optimal replacement problem", Ad- ${ }_{499}$ vances in Applied Probability, pp. 479-482, 1988.

[Yang et al.,2006] Yang, J., Zhao, J., Liao, D., Gregersen, H.: "Biomechan- ${ }^{501}$ ical properties of the layered oesophagus and its remodelling in experi- 502 mental type-1 diabetes", Journal of biomechanics, 39(5), pp. 894-904, 503 2006. 